Bangladesh J. Plant Taxon. 24(1): 107-116, 2017 (June)

\title{
PLANT DIVERSITY OF SONADIA ISLAND - AN ECOLOGICALLY CRITICAL AREA OF SOUTH-EAST BANGLADESH
}

\author{
M.S. Arefin, M.K. Hossain $^{1}$ And M. AKhter Hossain \\ Institute of Forestry and Environmental Sciences, University of Chittagong, \\ Chittagong 4331, Bangladesh
}

Keywords: Plant Diversity; Ecologically Critical Area; Sonadia Island; Mangroves.

\begin{abstract}
The study focuses the plant diversity in different habitats, status and percentage distribution of plants in Sonadia Island, Moheshkhali, Cox's Bazar of Bangladesh. A total of 138 species belonging to 121 genera and 52 families were recorded and the species were categorised to tree (56 species), shrub (17), herb (48) and climber (17). Poaceae represents the largest family containing 8 species belonging to 8 genera. Homestead vegetation consists of $78 \%$ species followed by roadside $(23 \%)$ and cultivated land (10\%), mangroves (9\%), sandy beaches (4\%) and wetland (1\%). The major traditional use categories were timber, food and fodder, fuel, medicine and fencing where maximum plant species (33\% of recorded) were traditionally being used for food and fodder.
\end{abstract}

\section{Introduction}

Sonadia Island at Moheshkhali of Cox's Bazar is situated in the southern-eastern coastal region of Bangladesh with partial regular inundations of saline water. The island covers an area of 10,298 hectares including coastal and mangrove plantations, salt production fields, shrimp culture firms, plain agriculture lands, human settlements etc. Ecosystem of this island was adversely affected due to increasing rate of anthropogenic disturbances. To protect the ecosystem of this island, it was declared as Ecologically Critical Area (ECA) in 1999 under section of the Bangladesh Environment Conservation Act, 1995 (MoEF, 2015). ECAs are ecologically defined areas or ecosystems affected adversely by the changes brought through human activities. This island is floristically composed of a number of mangrove and terrestrial plant species. The island is important not only as renewable resources but also as an essential in conservation of nature, wildlife, fish and environment of the island and the surrounding areas. The ECA needs special attention for environmental conservation in terms of both flora and fauna aspects. For this, a comprehensive list of the flora and fauna existing in Sonadia Island is essential. Moloney (2006) reported 60 vascular plants from Sonadia in the draft Sonadia Island ECA Conservation and Management Plan. There had been gradual changes in the ecological conditions due to increased anthropogenic interference. Since, no complete study was carried out throughout the period, it is completely unknown if any changes in the floristic composition of the critically endangered ecosystem has occurred in the last decade. Therefore, the present study was undertaken with the aim of assessing the plant resources of Sonadia Island, an ECA based on extensive field observations.

\section{Materials and Methods}

\section{Study area}

Sonadia Island is located in the far south-eastern corner of Bangladesh at $21^{\circ} \mathrm{N}$ and $91^{\circ} \mathrm{E}$, the site lies a few kilometers north of Teknaf Peninsula, north-west of Cox's Bazar town and is bounded by the Bay of Bengal on the West and East (Fig. 1).

${ }^{1}$ Corresponding author: Email: mkhossain2009@gmail.com

DOI: http://dx.doi.org/10.3329/bjpt.v24i1.33037 

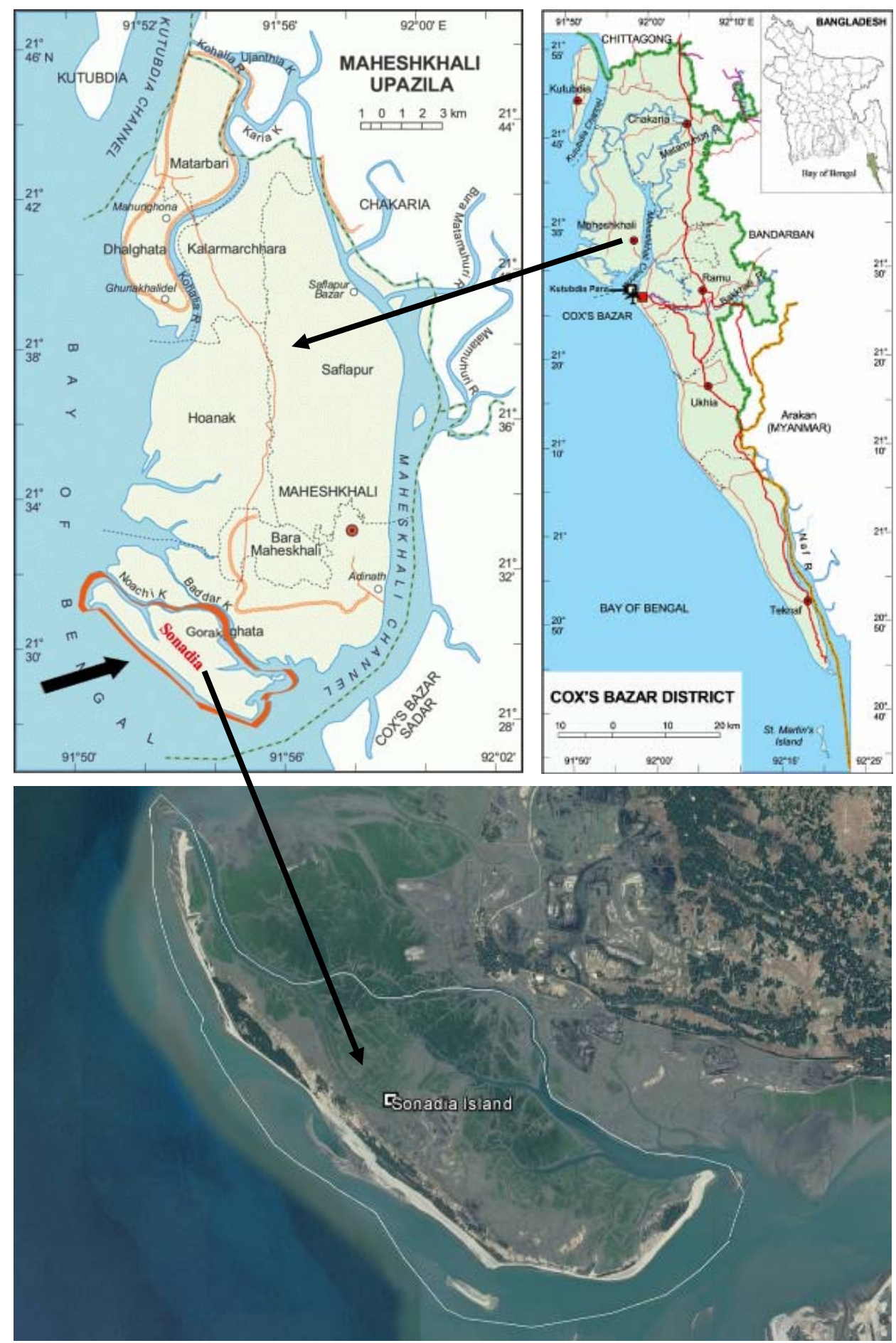

Fig. 1. Location of Sonadia island in Moheshkhali upazila of Cox’s Bazar district, Bangladesh. 
The Island is separated from the mainland by the Moheshkhali channel and from Moheshkhali Island by the Bara Canal. The soil of this area is the admixture of sand and clay in varying proportion. The soil of the North part is clay and is inundated by sea water. The entire soil condition of the South part is almost sandy (DoE, 1999). The whole island has a mild temperature and high humidity. The summer begins from March and continues till the beginning of June. The annual average temperature in Cox's Bazar is $34.8^{\circ} \mathrm{C}$ and a minimum of $16.1^{\circ} \mathrm{C}$. Sonadia Island is a gently sloping low-lying barrier island with an altitude range of 0-4 metres (DoE, 1999).

\section{Field visit, data collection and analysis}

A reconnaissance survey was conducted in the Sonadia island ECA prior to the field work to have a general idea of the site, topography, species composition, habitat condition and socioeconomic status of the local people. The flora study methods include key informant interview, reconnaissance survey, and field data collection through whole area survey, homestead plant survey, and focused group discussion from October, 2015 to August 2016. Field work was scheduled in such a way that enable plant observation and specimen collections of unknown plant species during the flowering and fruiting time of maximum number of species.

A total 9 foot trails of different length (3-7 km each) in two villages (Purbo para and Passchim para) and 9 boat journey through the surrounding canals, rivers and sea shore (total 33 $\mathrm{km}$ (approx.) were made to record the flora of homesteads and mangrove forests of Sonadia island. Survey was continued until occurrence of new species. The observed plant species were identified and recorded in the field. Habitat and habit form were also recorded. Herbarium specimen of rare and unidentified plant samples with fertile material (flower, fruit and seed) were collected and prepared for identification after necessary processing. Plant specimens with only vegetative part were also collected for herbarium preparation in case of unavailability of fertile materials. Photographs of the characteristic plant species from suitable projection were taken to keep a digital record of morphological features of the plants. Along with verification of the local names, local use of the recorded plants was explored through focused group discussion in the two villages of Sonadia Island.

Herbarium specimens were identified by consultation with voucher specimens and taxonomists of Bangladesh Forest Research Institute as well as recognized references, viz. Prain (1903); Heinig (1925); Siddiqui et al. (2007) and Ahmed et al. (2008). The identified taxa were arranged alphabetically with species names.

\section{Results}

\section{Floristic composition}

A total of 138 plant species belonging to 121 genera and 52 families were identified from the Sonadia island (Table 1). Among the recorded 138 species, Poaceae appeared as the largest family with 8 species under 8 genera followed by Cucurbitaceae (7 genera and 8 species), and Mimosaceae (8 species and 6 genera) (Fig. 2). Most of the families (28 nos.) were represented by only 1 species each (Table 1 ).

\section{Growth (habit) forms of the plants}

The recorded flora of Sonadia Island is grouped under tree, shrubs, herbs and climbers growth (habit) forms. Trees constitute the major category (56 species) of plant species followed by herbs (48 species), shrubs (17 species), and climbers (17 species) (Fig. 3). Number of tree species in Mimosaceae was maximum (5 genera and 7 species), whereas shrubs were maximum in Verbenaceae (3 genera and 4 species). In case of herbs and climbers Amaranthaceae (4 genera and 7 species) and Cucurbitaceae ( 7 genera and 8 species) were represented by maximum species respectively. 
Table 1. List of plant species recorded from Sonadia Island of Bangladesh.

\begin{tabular}{|c|c|c|c|c|c|}
\hline $\mathrm{SN}$ & Scientific name & Local name & Family name & Habit & Habitat \\
\hline 1 & $\begin{array}{l}\text { Acacia auriculiformis A. Cunn. ex Benth. } \\
\text { \& Hook. }\end{array}$ & Akashmoni & Mimosaceae & $\mathrm{T}^{*}$ & $\begin{array}{l}\text { Homestead, } \\
\text { Roadside }\end{array}$ \\
\hline 2 & Abelmoschus esculentus (L.) Moench & Vandi & Malvaceae & $\mathrm{H}$ & Cultivated \\
\hline 3 & Acacia farnesiana (L.) Willd. & Bilati Babla & Mimosaceae & $\mathrm{T}$ & Homestead \\
\hline 4 & Acanthus ilicifolius L. & Hargoza & Acanthaceae & S & Mangrove \\
\hline 5 & Aegialitis rotundifolia Roxb. & Nunia gach & Plumbaginaceae & S & Mangrove \\
\hline 6 & Albizia lebbeck (L.) Benth. & Kala koroi & Mimosaceae & $\mathrm{T}$ & Homestead \\
\hline 7 & Albizia procera (Roxb.) Benth. & Sada koroi & Mimosaceae & $\mathrm{T}$ & Homestead \\
\hline 8 & Alocasia macrorrhizos (L.) G. Don & Mankachu & Amaranthaceae & $\mathrm{H}$ & Homestead \\
\hline 9 & $\begin{array}{l}\text { Alternanthera philoxeroides (Mart.) } \\
\text { Griseb. }\end{array}$ & Helencha & Amaranthaceae & $\mathrm{H}$ & $\begin{array}{l}\text { Cultivated, } \\
\text { Roadside }\end{array}$ \\
\hline 10 & $\begin{array}{l}\text { Alternanthera sessilis (L.) R. Br. ex } \\
\text { Roem. \& Schult. }\end{array}$ & Saci Shak & Amaranthaceae & $\mathrm{H}$ & Cultivated \\
\hline 11 & Amaranthus spinosus L. & Katashak & Amaranthaceae & $\mathrm{H}$ & Homestead \\
\hline 12 & Amaranthus tricolor $\mathrm{L}$. & Lalshak & Amaranthaceae & $\mathrm{H}$ & $\begin{array}{l}\text { Homestead, } \\
\text { Cultivated }\end{array}$ \\
\hline 13 & Amaranthus viridis $\mathrm{L}$. & Datashak & Amaranthaceae & $\mathrm{H}$ & $\begin{array}{l}\text { Homestead, } \\
\text { Roadside }\end{array}$ \\
\hline 14 & Anacardium occidentale L. & Kajubadam & Anacardiaceae & $\mathrm{T}$ & Homestead \\
\hline 15 & Areca catechu L. & Supari & Arecaceae & $\mathrm{T}$ & Homestead \\
\hline 16 & Argyreia capitiformis (poir.) Oostr. & Voga Lata & Convolvulaceae & $\mathrm{C}$ & Roadside \\
\hline 17 & Artocarpus heterophyllus Lamak. & Kathal & Moraceae & $\mathrm{T}$ & Homestead \\
\hline 18 & Asystasia gangetica (L.) T. Anders. & & Acanthaceae & $\mathrm{H}$ & Roadside \\
\hline 19 & Averrhoa carambola L. & Kamranga & Averrhoaceae & $\mathrm{T}$ & Homestead \\
\hline 20 & Avicennia alba Blume. & Sada Baen & Avicenniaceae & $\mathrm{T}$ & Mangrove \\
\hline 21 & Avicennia marina (Forsk.) Vierh. & Moriccha Baen & Avicenniaceae & $\mathrm{T}$ & Mangrove \\
\hline 22 & Avicennia officinalis L. & Kalo Baen & Avicenniaceae & $\mathrm{T}$ & Mangrove \\
\hline 23 & Azadirachta indica A.Juss. & Neem & Meliaceae & $\mathrm{T}$ & Homestead \\
\hline 24 & Bambusa vulgaris Schrad.ex Wendl. & Baijja Bans & Poaceae & $\mathrm{T}$ & Homestead \\
\hline 25 & Basella rubra L. & Poi Shak & Basellaceae & $\mathrm{C}$ & Homestead \\
\hline 26 & Benincasa hispida (Thunb.) Cogn. & Chal Kumra & Cucurbitaceae & $\mathrm{C}$ & Homestead \\
\hline 27 & Blumea lacera (Burm.f.) & Kukur Muta & Asteraceae & $\mathrm{H}$ & Roadside \\
\hline 28 & Brassica juncea (L.) Czern. & Rai Sorisa & Brassicaceae & $\mathrm{H}$ & Homestead \\
\hline 29 & Calotropis procera (Ait.) R. Br. & Akanda & Asclepiadaceae & $\mathrm{T}$ & Homestead \\
\hline 30 & Canavalia virosa (Roxb.) Wight \& Arn. & Kalo Shim & Fabaceae & $\mathrm{H}$ & Homestead \\
\hline 31 & Capsicum frutescens L. & Morich & Solanaceae & $\mathrm{H}$ & $\begin{array}{l}\text { Homestead, } \\
\text { Cultivated }\end{array}$ \\
\hline 32 & Carica papaya L. & Pepe & Caricaceae & S & Homestead \\
\hline 33 & Carissa carandas L. & Koromcha & Apocynaceae & $\mathrm{S}$ & Homestead \\
\hline 34 & Cassia fistula $\mathrm{L}$. & Sonalu & Caesalpiniaceae & $\mathrm{T}$ & Homestead \\
\hline 35 & Casuarina equisetifolia Forst. & Jau & Casuarinaceae & $\mathrm{T}$ & $\begin{array}{l}\text { Sandy } \\
\text { beach, } \\
\text { Roadside }\end{array}$ \\
\hline
\end{tabular}




\begin{tabular}{|c|c|c|c|c|c|}
\hline SN & Scientific name & Local name & Family name & Habit & Habitat \\
\hline 36 & Catharanthus roseus (L.) G.Don & Nayantara & Apocynaceae & $\mathrm{H}$ & Homestead \\
\hline 37 & Ceiba pentandra (L.) Gaertn. & Burma Simul & Bombacaceae & $\mathrm{T}$ & Homestead \\
\hline 38 & Cicca acida (L.) Merr. & Orboroi & Euphorbiaceae & $\mathrm{T}$ & Homestead \\
\hline 39 & $\begin{array}{l}\text { Citrus aurantifolia (Christm. \& Panzer) } \\
\text { Swingle }\end{array}$ & Lebu & Rutaceae & $\mathrm{T}$ & Homestead \\
\hline 40 & Citrus grandis (L.) Osbeck & Jambura & Rutaceae & $\mathrm{T}$ & Homestead \\
\hline 41 & $\begin{array}{l}\text { Citrullus lanatus (Thunb.) Matsum. \& } \\
\text { Nakai }\end{array}$ & Tormuj & Cucurbitaceae & $\mathrm{C}$ & Cultivated \\
\hline 42 & Clerodendrum inerme (L.) Gaertn. & Bonjui & Verbenaceae & S & Roadside \\
\hline 43 & Cocos nucifera L. & Narikel & Arecaceae & $\mathrm{T}$ & Homestead \\
\hline 44 & Colocasia esculenta (L.) Schott & Kachu & Araceae & $\mathrm{H}$ & Homestead \\
\hline 45 & Commelina benghalensis L. & Kanchira & Commelinaceae & $\mathrm{H}$ & Cultivated \\
\hline 46 & Corypha umbraculifera L. & & Arecaceae & $\mathrm{T}$ & Homestead \\
\hline 47 & Crotalaria juncea L. & Junjuni & Fabaceae & $\mathrm{H}$ & Roadside \\
\hline 48 & Cucumis melo L. & Bangi & Cucurbitaceae & $\mathrm{C}$ & Cultivated \\
\hline 49 & Cucumis sativus L. & Khira & Cucurbitaceae & $\mathrm{C}$ & Cultivated \\
\hline 50 & Cucurbita maxima Duch. ex Lamk. & Misti Kumra & Cucurbitaceae & $\mathrm{C}$ & $\begin{array}{l}\text { Homestead, } \\
\text { Cultivated }\end{array}$ \\
\hline 51 & Curcuma longa L. & Halud & Zingiberaceae & $\mathrm{H}$ & Homestead \\
\hline 52 & Cynodon dactylon (L.) Pers. & Durbagass & Poaceae & $\mathrm{H}$ & Roadside \\
\hline 53 & Cyperus javanicus Houtt. & Kucha & Сyperaceae & $\mathrm{H}$ & Roadside \\
\hline 54 & Dalbergia spinosa Roxb. & Churilla kanta & Fabaceae & $\mathrm{C}$ & Mangrove \\
\hline 55 & Delonix regia Rafin. & Krishnachura & Caesalpiniaceae & $\mathrm{T}$ & Homestead \\
\hline 56 & $\begin{array}{l}\text { Dendrocalamus giganteus Wall. ex } \\
\text { Munro }\end{array}$ & Budhum bans & Poaceae & $\mathrm{T}$ & Homestead \\
\hline 57 & Dioscorea bulbifera L. & Pagla alu & Dioscoreaceae & $\mathrm{S}$ & Homestead \\
\hline 58 & Eclipta alba (L.) Hassk. & Kesaraj & Asteraceae & $\mathrm{H}$ & Roadside \\
\hline 59 & Elaeis guineensis Jacq. & Oil Palm & Arecaceae & $\mathrm{T}$ & Homestead \\
\hline 60 & Erythrina fusca Lour. & Kata Mandar & Fabaceae & $\mathrm{T}$ & Homestead \\
\hline 61 & Eucalyptus camaldulensis Dehnh. & Euclyptus & Myrtaceae & $\mathrm{T}$ & Homestead \\
\hline 62 & Eupatorium odoratum L. & Assam Gach & Asteraceae & $\mathrm{H}$ & Cultivated \\
\hline 63 & Excoecaria agallocha $\mathrm{L}$. & Gewa & Euphorbiaceae & $\mathrm{T}$ & Mangrove \\
\hline 64 & Ficus benghalensis L. & Bot & Moraceae & $\mathrm{T}$ & Homestead \\
\hline 65 & Garuga pinnata Roxb. & Bhadi & Burseraceae & $\mathrm{T}$ & Homestead \\
\hline 66 & Gmelina arborea Roxb. & Gamar & Verbenaceae & $\mathrm{T}$ & Homestead \\
\hline 67 & Hedyotis corymbosa (L.) Lam. & Khetpapra & Rubiaceae & $\mathrm{H}$ & $\begin{array}{l}\text { Roadside, } \\
\text { Cultivated }\end{array}$ \\
\hline 68 & Heliotropium curassavicum L. & Hatisur & Boraginaceae & S & Mangrove \\
\hline 69 & Heliotropium indicum L. & Hatisur & Boraginaceae & $\mathrm{H}$ & Roadside \\
\hline 70 & Hibiscus rosa-sinensis L. & Joba & Malvaceae & $\mathrm{S}$ & Homestead \\
\hline 71 & Hopea odorata Roxb. & Telsur & Dipterocarpaceae & $\mathrm{T}$ & Homestead \\
\hline 72 & Hyptis suaveolens (L.) Poit. & Tokma & Lamiaceae & S & Roadside \\
\hline 73 & Imperata cylindrica (L.) P. Beauv. & Chan & Poaceae & $\mathrm{H}$ & Roadside \\
\hline
\end{tabular}




\begin{tabular}{|c|c|c|c|c|c|}
\hline SN & Scientific name & Local name & Family name & Habit & Habitat \\
\hline 74 & Ipomea batatas (L.) Lam. & Misti alu & Convolvulaceae & $\mathrm{C}$ & $\begin{array}{l}\text { Homestead, } \\
\text { Cultivated }\end{array}$ \\
\hline 75 & Ipomea pes-caprae (L.) R. Br. & Sagorlata & Convolvulaceae & $\mathrm{C}$ & Sandy beach \\
\hline 76 & Ipomoea aquatica Forsk. & Kolmi Shak & Convolvulaceae & $\mathrm{C}$ & Homestead \\
\hline 77 & Ipomoea fistulosa Mart. ex Choisy & Dolkolomi & Convolvulaceae & S & Roadside \\
\hline 78 & Jatropha curcas L. & Baghverenda & Euphorbiaceae & S & Roadside \\
\hline 79 & Justicia gendarussa Burm. f. & Jagmodon & Acanthaceae & $\mathrm{H}$ & Roadside \\
\hline 80 & Lablab purpureus (L.) Sweet & Sheem & Fabaceae & $\mathrm{C}$ & Homestead \\
\hline 81 & Lagenaria vulgaris Seringe & Lao & Cucurbitaceae & $\mathrm{C}$ & Homestead \\
\hline 82 & Lagerstroemia speciosa (L.) Pers. & Jarul & Lythraceae & $\mathrm{T}$ & Roadside \\
\hline 83 & Lannea coromandelica (Houtt.) Merr. & Bhadi & Anacardiaceae & $\mathrm{T}$ & Homestead \\
\hline 84 & Lantana camara L. & Moggula & Verbenaceae & S & $\begin{array}{l}\text { Homestead, } \\
\text { Roadside }\end{array}$ \\
\hline 85 & $\begin{array}{l}\text { Launaea sarmentosa (Wild.) Sch. Bip. ex } \\
\text { Kantze }\end{array}$ & & Asteraceae & $\mathrm{H}$ & Roadside \\
\hline 86 & Lawsonia inermis L. & Mendi & Lythraceae & S & Homestead \\
\hline 87 & Leucaena leucocephala (Lam.) de Wit. & Ipil-Ipil & Mimosaceae & $\mathrm{T}$ & Homestead \\
\hline 88 & Leucas aspera (willd.) Link. & Shetodhrona & Lamiaceae & $\mathrm{H}$ & Roadside \\
\hline 89 & Leucas cephalotes (Roth) Spreng. & Bara-halkus & Lamiaceae & $\mathrm{H}$ & Roadside \\
\hline 90 & Lindernia ciliata (Colsm.) Pennell & Bhui & Scrophulariaceae & $\mathrm{T}$ & Roadside \\
\hline 91 & Ludwigia adscendens (L.) Hara & Kesra-dum & Onagraceae & $\mathrm{H}$ & Roadside \\
\hline 92 & Luffa cylindrica M. Roem. & Dundul & Cucurbitaceae & $\mathrm{C}$ & Roadside \\
\hline 93 & Lumnitzera racemosa Willd. & Kirpa & Combretaceae & $\mathrm{T}$ & Mangrove \\
\hline 94 & Lycopersicon esculentum Mill. & Tomato & Solanaceae & $\mathrm{H}$ & $\begin{array}{l}\text { Homestead, } \\
\text { Cultivated }\end{array}$ \\
\hline 95 & Mangifera indica L. & Aam & Anacardiaceae & $\mathrm{T}$ & Homestead \\
\hline 96 & Mimosa pudica L. & Lojjaboti & Mimosaceae & $\mathrm{H}$ & Roadside \\
\hline 97 & Moringa oleifera Lamk. & Shajna & Moringaceae & $\mathrm{T}$ & Homestead \\
\hline 98 & Musa paradisiaca L. & Kola & Musaceae & $\mathrm{H}$ & Homestead \\
\hline 99 & Neolamarckia cadamba (Roxb.) Bosser. & Kadam & Rubiaceae & $\mathrm{T}$ & Homestead \\
\hline 100 & Opuntia dillenii Haw. & Foni Monsha & Cactaceae & $\mathrm{C}$ & Homestead \\
\hline 101 & Oryza sativa $\mathrm{L}$ & Dhan & Poaceae & $\mathrm{H}$ & Cultivated \\
\hline 102 & Oxystelma secamone (L.) Karst. & Dudhia kata & Asclepiadaceae & $\mathrm{H}$ & Roadside \\
\hline 103 & Pandanus fascicularis Lamk. & Keyakata & Pandanaceae & $\mathrm{T}$ & Sandy beach \\
\hline 104 & Pandanus foetidus Roxb. & Keyakata & Pandanaceae & S & Sandy beach \\
\hline 105 & Paspalum vaginatum Sw. & & Poaceae & $\mathrm{H}$ & Cultivated \\
\hline 106 & Passiflora foetida L. & Jumka lata & Passifloraceae & $\mathrm{C}$ & Homestead \\
\hline 107 & Phoenix sylvestris (L.) Roxb. & Deshi Khejur & Arecaceae & $\mathrm{T}$ & Homestead \\
\hline 108 & Pithecellobium dulce (Roxb.) Benth. & Jilapi & Mimosaceae & $\mathrm{T}$ & Homestead \\
\hline 109 & Porteresia coarctata (Roxb.) Tateoka & Urigrass & Poaceae & $\mathrm{H}$ & $\begin{array}{l}\text { Mangrove } \\
\text { meadow }\end{array}$ \\
\hline 110 & Portulaca oleracea L. & Nuinnashak & Portulacaceae & $\mathrm{H}$ & $\begin{array}{l}\text { Mangrove } \\
\text { meadow }\end{array}$ \\
\hline 111 & Psidium guajava L. & Payara & Myrtaceae & $\mathrm{T}$ & Homestead \\
\hline
\end{tabular}




\begin{tabular}{|c|c|c|c|c|c|}
\hline $\mathrm{SN}$ & Scientific name & Local name & Family name & Habit & Habitat \\
\hline 112 & $\begin{array}{l}\text { Psilotrichum ferrugineum (Roxb.) Moq.- } \\
\text { Tand. }\end{array}$ & $\begin{array}{l}\text { Khetapada } \\
\text { Shak }\end{array}$ & Amaranthaceae & $\mathrm{H}$ & $\begin{array}{l}\text { Homestead, } \\
\text { Roadside }\end{array}$ \\
\hline 113 & Raphanus sativus L. & Mula & Brassicaceae & $\mathrm{H}$ & Cultivated \\
\hline 114 & Ricinus communis L. & Varenda & Euphorbiaceae & $\mathrm{T}$ & Homestead \\
\hline 115 & Samanea saman (Jacq.) Merr. & Raintree & Mimosaceae & $\mathrm{T}$ & Homestead \\
\hline 116 & Senna tora (L.) Roxb. & Terasena & Caesalpiniaceae & $\mathrm{H}$ & Roadside \\
\hline 117 & Sida cordifolia L. & Berela & Malvaceae & $\mathrm{H}$ & Homestead \\
\hline 118 & Solanum melongena $\mathrm{L}$. & Begun & Solanaceae & $\mathrm{H}$ & Homestead \\
\hline 119 & Sonneratia apetala Buch.-Ham. & Keora & Sonneratiaceae & $\mathrm{T}$ & Mangrove \\
\hline 120 & Spinacia oleracea L. & Palon Shak & Chenopodiaceae & $\mathrm{H}$ & Homestead \\
\hline 121 & Spondias pinnata (L. f.) Kurz. & Amra & Anacardiaceae & $\mathrm{T}$ & Homestead \\
\hline 122 & Streblus asper Lour. & Sheora & Moraceae & $\mathrm{T}$ & Homestead \\
\hline 123 & Suaeda maritima (L.) Dumort. & & Chenopodiaceae & $\mathrm{H}$ & Roadside \\
\hline 124 & Swietenia mahagoni Jacq. & Mahogoni & Meliaceae & $\mathrm{T}$ & Homestead \\
\hline 125 & Synedrella nodiflora (L.) Gaertn. & Not known & Asteraceae & $\mathrm{H}$ & Roadside \\
\hline 126 & Syzygium fruticosum DC. & Putijam & Myrtaceae & $\mathrm{T}$ & Homestead \\
\hline 127 & Tamarindus indica L. & Tentul & Caesalpiniaceae & $\mathrm{T}$ & Homestead \\
\hline 128 & Tamarix gallica L. & Nona jau & Tamaricaceae & S & Mangrove \\
\hline 129 & Tephrosia purpurea (L.) Pers. & Bon-neel & Fabaceae & $\mathrm{H}$ & Cultivated \\
\hline 130 & Terminalia arjuna (Roxb. Ex DC.) & Arjun & Combretaceae & $\mathrm{T}$ & Roadside \\
\hline 131 & Terminalia catappa $\mathrm{L}$. & Kat Badam & Combretaceae & $\mathrm{T}$ & Homestead \\
\hline 132 & Thevetia peruviana (Pers.) K. Schum. & Halde Karabi & Apocynaceae & $\mathrm{T}$ & Homestead \\
\hline 133 & Thysanolaena maxima (Roxb.) O. Kuntze & Jahruful & Poaceae & $\mathrm{H}$ & Homestead \\
\hline 134 & Trichosanthes anguina L. & Chichinga & Cucurbitaceae & $\mathrm{C}$ & $\begin{array}{l}\text { Cultivated, } \\
\text { Homestead }\end{array}$ \\
\hline 135 & Typha domingensis (Pars.) ex Steud. & Hogla & Typhaceae & $\mathrm{H}$ & Wetland \\
\hline 136 & Vitex negundo L. & Nil Nishinda & Verbenaceae & $\mathrm{S}$ & $\begin{array}{l}\text { Sandy beach, } \\
\text { Roadside }\end{array}$ \\
\hline 137 & Vitex trifolia L. f. & Nishinda & Verbenaceae & $\mathrm{S}$ & $\begin{array}{l}\text { Sandy beach, } \\
\text { roadside }\end{array}$ \\
\hline 138 & Ziziphus mauritiana Lamk. & Boroi & Rhamnaceae & $\mathrm{T}$ & Homestead \\
\hline
\end{tabular}

[* T- Tree, S-Shrub, H-Herb, C-Climber]

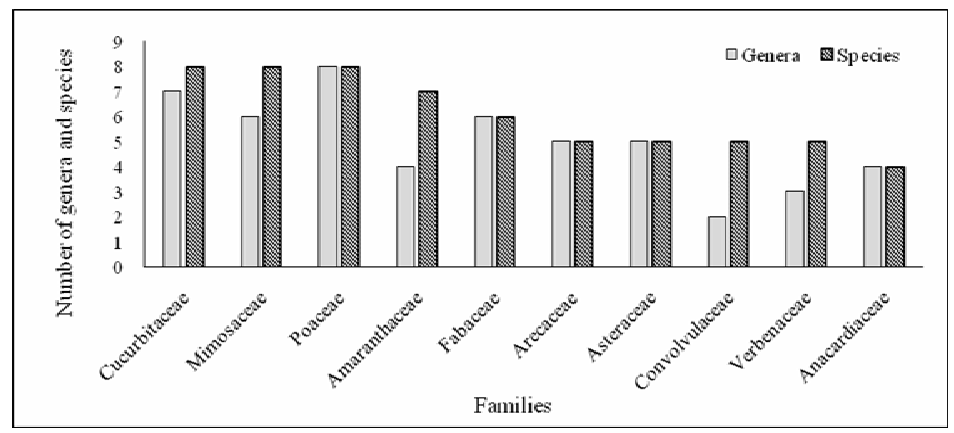

Fig. 2. Number of species belonging to dominant Family in Sonadia Island. 


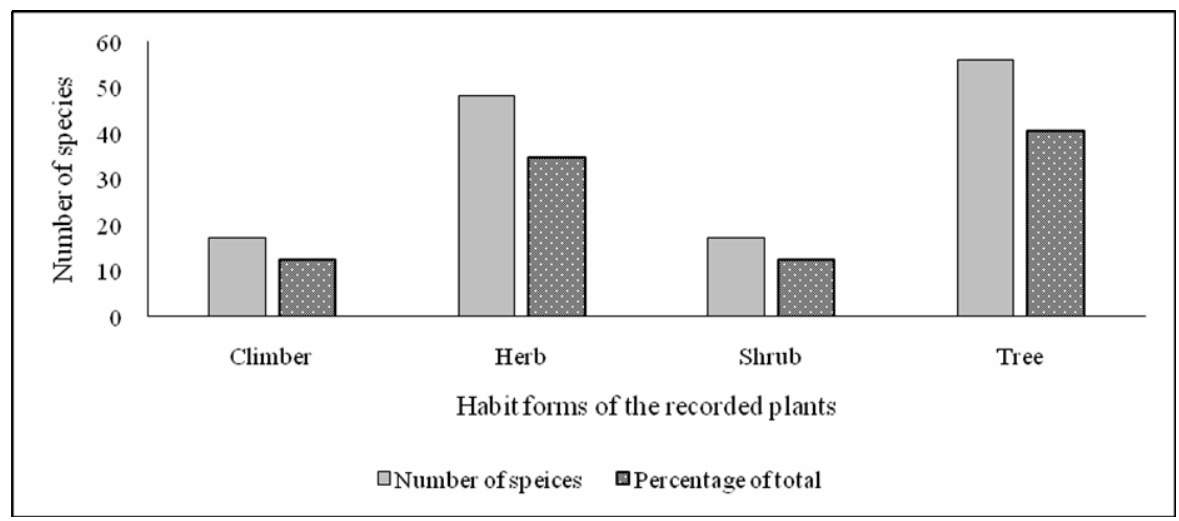

Fig. 3. Number of species belonging to habit form in Sonadia island.

\section{Major plant habitats in Sonadia Island}

The Sonadia Island supports vegetation growing in 6 broad categories of habitats including sand dunes or sandy beach area, homestead, mangrove, mangrove meadow, bounds or foot trail or roadside and cultivated land. Homestead represented 78 species constituting 53\% of total species followed by $23 \%$ in roadside, $10 \%$ in cultivation firms, $9 \%$ in $10 \%$ in cultivation firms, $9 \%$ in mangrove, and $1 \%$ in wetland. Plants common in the sand dunes constitute $4 \%$ of total are species, in particular Ipomea pes-caprae, Vitex trifolia, Pandanus foetidus and Casuarina equisetifolia. Plants commonly occurring in the homesteads are Acacia auriculiformis, Cocos nucifera and Eucalyptus camaldulensis etc. Avicennia officinalis, Avicennia alba and Acanthus illicifolius appeared as very common in the natural mangrove forest, whereas in the plantations Casuarina equisetifolia, Eucalyptus camaldulensis, Acacia auriculiformis and Sonneratia apetala were commonly found.

\section{Traditional uses of the recorded plants}

Knowledge about the various uses of the available plants was gained through conversations made with the local peoples living within the island. Traditional use of the recorded plants indicate that most of the plants (33\%) have food value as fruit, flower, seed and different parts of those plants are edible in raw or after processing. Plants also used substantially as fuel wood (18\%), timber (11\%), biological fence (9\%), medicine (7\%) etc. It is found that many medicinal plants, their medicinal values and uses are not known to local people. Plants that provide fodder, oils, weeds etc. are grouped under miscellaneous category which constitutes $11 \%$ of all recorded plant species.

\section{Discussion}

The study reveals that Sonadia island currently harbours 138 plant species (tree 56, shrubs 17, herbs 48, climbers 17) that belong to 111 genera and 55 families which is higher in comparison to Moloney (2006) that recorded only 60 vascular plants from Sonadia island (14 trees, 8 shrubs, 27 herbs and 11 climbers species). In the first report on the angiospermic flora of this land (Khan et al., 1977) the number of species was for less. According to the people living in the island, vegetation coverage in mangrove forest was dense. The findings conform with the reports of Thompson and Islam (2010) who indicated 144 angiospermic plants from Saint Martin's Island of Cox`s Bazar. Sandwip, another island of Bangladesh harbours much higher plants (438 vascular 
plants) due to its comparatively larger area coverage and varied households with diversified domestic flora (Sajib et al., 2016). The floristic records of different island's of Bangladesh also reported 149 species from Moheshkhali (Huq and Khan, 1984), 151 species from the same island (Rashid et al., 2000), 91 species from Kutubdia island (Huq 1986), 98 plant species from Hatiya island (Huq 1988) and 37 species from Nijhum Dwip (Khan et al., 1985) and 152 species from Nijhum Dwip (Uddin et al., 2015).

The presence of some exotic tree species, i.e., Acacia auriculiformis, Swietenia mahagoni and Eucalyptus camaldulensis species was due to the plantations conducted by Bangladesh Forest Department and the local people. Major area of the island is occupied by natural mangroves, but encroachment is becoming a serious concern because of the conversion of forest lands to salt bed and shrimp cultivation. Jhau, the successful species in the sandy beaches of Cox's Bazar (Hossain, 2010)) is also promising in Sonadia Island but illegal felling is a common threat in the island).

\section{Acknowledgements}

The authors are grateful to the Research Cell authority of University of Chittagong for providing funds for field works. We are also grateful to the officers and field staffs of Chittagong Coastal Forest Division, Bangladesh Forest Department for helping in the field work. Thanks are due to Taxonomists of Forest Botany Division, BFRI and Dr. Mohammed Yusuf, Ex-Director of BCSIR for their supports in identification of the plant samples.

\section{References}

Ahmed, Z.U., Begum, Z.N.T., Hassan, M.A., Khondker, M., Kabir, S.M.H., Ahmad, M., Ahmed, A.T.A., Rahman, A.K.A. and Haque, E.U. (Eds). 2008. Encyclopedia of Flora and Fauna of Bangladesh, vol. 512. Asiatic Society of Bangladesh, Dhaka.

DoE (Department of Environment). 1999. GIS and Cartographic Services - Final Report, Pre-Investment Facility Study: Coastal and Wetland Biodiversity Management Project (Project BGD/94/G41), Dhaka, Bangladesh.

Heinig, R.L. 1925. List of Plants of Chittagong Collectorate and Hill Tracts. Darjeeling, India, 84 pp.

Hossain, M.K. 2010. Casuarina equisetifolia- a promising species for green belt project of coastal and offshore islands of Bangladesh. In: Zhong, C., Pinyopusarerk, K., Kalinganire, A. and Franche C. (eds.), Improving Smallholder Livelihoods Through Improved Casuarina Productivity: Proceedings of the $4^{\text {th }}$ International Casuarina Workshop, Haikou, China 21-25 March 2010. pp. 200-206.

Huq, A.M. 1986. Preliminary studies on the anthropogenic flora of Kutubdia Island in Bangladesh. J Asiatic Soc. Bangladesh (Sci.) 12: 59-70.

Huq, A.M. 1988. A Preliminary taxonomic report on the Angiospermic flora of Hatia Islan (Noakhali district) (Dicotyledons). Bull. Bangladesh Nat. Herb., Dhaka 1: 1-10.

Huq, A.M. and Khan, M.S. 1984. A preliminary taxonomic report on the angiospermic flora of Maheshkhali Island-1 (Dicotyledons). Dhaka Univ. Stud. B 32: 19-31.

Khan, M.S., Huq, M.A. Rahman, M.M. and Hassan, M.A. 1977. A preliminary report on the angiospermic flora of Sonadia island, Bangladesh. J. Asit. Sco. Bangladesh 3(1): 125-126.

Khan, M.S., Huq, A.M. and Rahman, M.M. 1985. Studies on the angiospermic flora of Nijhum Dwip (Char Osman) in the Bay of Bengal. Dhaka Univ. Stud. B 33: 145-151.

MoEF (Ministry of Environment and Forests) 2015. Fifth National Report to the Convention on Biological Diversity. Government of the People's Republic of Bangladesh, Dhaka, 164 pp.

Moloney, L. 2006. Coastal and Wetland Biodiversity Management Plan BGD/ 99/ G31 Sonadia Island ECA Draft Conservation Management Plan.

Prain, D. 1903 (Reprinted.1981). Bengal Plants. Calcutta, 1: 120 pp. 
Rashid, M.H., Rahman, E. and Rahman, M.A. 2000. Additions to the angiospermic flora of the Moheskhali island. Cox’s Bazar. Bangladesh J Plant Taxon 7: 43-63.

Sajib, N.H., Uddin, S.B. and Islam, M.S. 2016. Vascular plant diversity and their distribution pattern in Sandwip Island, Chittagong, Bangladesh. J Biodivers Manage Forestry 5: 2

Siddiqui, K.U., Islam, M.A., Ahmed, Z.U., Begum, Z.N.T., Hassan, M.A., Khondker, M., Rahman, M.M., Kabir, S.M.H., Ahmed, M., Ahmed, A.T.A., Rahman, A.K.A. and Haque, E.U. (eds). 2007. Encyclopedia of Flora and Fauna of Bangladesh, Vol. 11. Angiosperms: (Agavaceae - Najadaceae). Asiatic Society of Bangladesh, pp. 399.

Thompson, P.M. and Islam, M.A. (Eds.). 2010. Environmental Profile of St. Martin's Island, United Nations Development Programme, Dhaka. Washington, DC: Island Press, pp. 112-117.

Uddin, M.Z., Kibria, M.G. and Hassan, M.A. 2015. Assessment of angiosperm plant diversity of Nijhum Dweep. Bangladesh J. Asiat. Soc. Bangladesh, Sci. 41(1): 19-52.

(Manuscript received on 6 February 2017; revised on 11 May 2017) 